\title{
Relation between Dielectric and Low-Frequency Raman Spectra of Hydrogen-Bond Liquids
}

\author{
Toshiko Fukasawa, ${ }^{1}$ Takaaki Sato, ${ }^{2, *}$ Junji Watanabe, ${ }^{3}$ Yoshimasa Hama, ${ }^{1}$ Werner Kunz, ${ }^{4}$ and Richard Buchner ${ }^{4, \dagger}$ \\ ${ }^{1}$ Advanced Research Institute for Science and Engineering, Waseda University, Okubo 3-4-1, Shinjuku-ku, Tokyo 169-8555, Japan \\ ${ }^{2}$ Division of Pure and Applied Physics, Faculty of Science and Engineering, Waseda University, Okubo 3-4-1, Shinjuku-ku, \\ Tokyo 169-8555, Japan \\ ${ }^{3}$ Graduate School of Frontier Biosciences, Osaka University, Yamadaoka 1-3, Suita-shi, Osaka, 565-0871, Japan \\ ${ }^{4}$ Institut für Physikalische und Theoretische Chemie, Universität Regensburg, D-93040 Regensburg, Germany
}

(Received 8 July 2005; published 3 November 2005)

\begin{abstract}
We analyzed the complex dielectric and Raman spectra of hydrogen-bond liquids in the microwave to terahertz frequency range. As for water and methanol, the high-frequency component of the dielectric spectrum, i.e., the small deviation from the principal Debye relaxation, clearly corresponds to the Raman spectrum. This indicates that the cooperative relaxation, accompanied by huge polarization fluctuation, is virtually not Raman active, whereas the faster processes reflect common microscopic dynamics. For ethylene glycol, the shape of the Raman spectrum also resembles that of the high-frequency deviation of the dielectric spectrum, but, additionally, a weak manifestation of the cooperative relaxation arising from quadrupolar conformers is detected.
\end{abstract}

DOI: 10.1103/PhysRevLett.95.197802

PACS numbers: 61.25.Em

Introduction. - It is well established that Raman scattering and infrared spectroscopy, which observe microscopic molecular dynamics, respectively, through the change of polarizability and total dipole moment, yield basically complementary information on intra- and intermolecular vibrations. We found that, in the case of dipolar aprotic liquids such as acetonitrile, acetone, and tetrahydrofuran (THF), the relaxation mode of the Raman susceptibility is well explained if the loss peak frequency in the dielectric spectrum is shifted by a factor of 3 toward higher frequencies. The oscillator components seen in the terahertz (THz) frequency range of both spectra appear at the same positions; see Fig. 1. This demonstrates that, for these nonhydrogen-bond liquids, both dynamic susceptibilities reflect the common molecular dynamics of rotational diffusion and the subsequent librational motions [1]. If the Raman spectra are plotted with proper relative intensities, the sequence of the intensities is in the order of the static dielectric constants; see Fig. 1(b). On the other hand, hydrogen-bond liquids, such as water and monohydric alcohols, exhibit pronounced complex dielectric and Raman spectra which differ considerably in shape in the microwave to sub-THz frequency range. The Raman intensities are far weaker than expected from the spectra of nonhydrogen-bonding dipolar liquids of similar dielectric constants. This suggests that Raman and dielectric dynamic susceptibilities now reflect different kinds of molecular dynamics.

A limited number of attempts were made to compare the first $(l=1)$ and second $(l=2)$ rank relaxation times, $\tau(l)$, of water [2-5]. The authors discussed a possible correspondence between $\tau(1)$ obtained from THz-time domain spectroscopy (THz-TDS) and $\tau(2)$ data from NMR and optical-heterodyne detected, Raman-induced Kerr effect spectroscopy $[2,4,5]$. Nevertheless, the general relation between the first- and the second-rank dynamic susceptibilities of hydrogen-bond liquids remained unclear. The goal of this study is to work out a general rule that can consistently explain the interplay between the dielectric

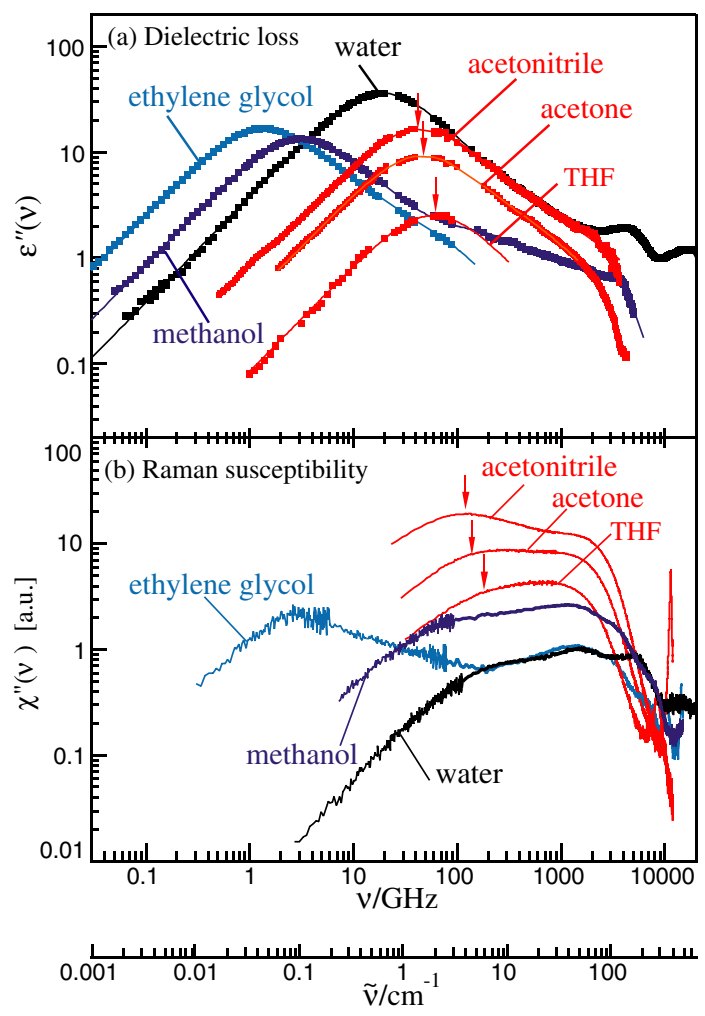

FIG. 1 (color online). (a) Dielectric loss $\epsilon^{\prime \prime}(\nu)$ and (b) Raman susceptibility $\chi^{\prime \prime}(\tilde{\nu})$ spectra of hydrogen-bond and dipolar aprotic liquids at $25^{\circ} \mathrm{C}$. For the nonhydrogen-bonding liquids, the arrows on the dielectric spectra indicate their loss peak frequencies, $\nu_{\text {diel }}$; those on the Raman spectra show $3 \nu_{\text {diel }}$. 
and Raman spectra of representative hydrogen-bond liquids and the underlying molecular mechanisms. This has been achieved by a direct comparison between these two dynamic susceptibilities in frequency domain.

Experimental. - The depolarized $(\mathrm{VH})$ low-frequency Raman spectra of hydrogen-bond liquids (water, methanol, and ethylene glycol) and polar liquids (acetonitrile, acetone, and tetrahydrofuran) were measured at $25^{\circ} \mathrm{C}$. A double-grating spectrometer (Jovin-Yvon U1000) with a resolution of $1.8 \mathrm{~cm}^{-1}$ was used for wave numbers $\tilde{\nu}>$ $2 \mathrm{~cm}^{-1}$, whereas for the hydrogen-bond liquids a Sandercock-type Fabry-Perot interferometer was employed at $\tilde{\nu} \leq 4 \mathrm{~cm}^{-1}$ to get highly reliable low-frequency data [6]. For the interferometer a resolution of $0.04 \mathrm{~cm}^{-1}$ was achieved for water and methanol and $0.0042 \mathrm{~cm}^{-1}$ for ethylene glycol. The Raman intensity $I(\tilde{\nu})$ was reduced into the imaginary part of the complex dynamic susceptibility, $\chi^{\prime \prime}(\tilde{\nu})$. We also measured the complex dielectric spectra $\hat{\epsilon}(\nu)=\epsilon^{\prime}(\nu)-i \epsilon^{\prime \prime}(\nu)$ of these liquids at $25^{\circ} \mathrm{C}$. Time domain reflectometry [7] was used in the frequency region of $0.02 \leq \nu / \mathrm{GHz} \leq 25$ and waveguide interferometry in $13 \leq \nu / \mathrm{GHz} \leq 89[8,9]$. These spectra were combined with published THz-TDS [3,10] and far-infrared (FIR) data [11,12] interpolated to the investigated temperature at each $\nu$, achieving the maximum frequencies of $\nu_{\max }=18 \mathrm{THz}$ for water and $5 \mathrm{THz}$ for methanol, whereas $\nu_{\max }$ for ethylene glycol is limited to $89 \mathrm{GHz}$.

Results and discussions. - The main contribution to the dielectric spectrum of water, the so-called $\tau_{1}$ relaxation, perfectly obeys a Debye relaxation function up to several tens of gigahertz. Then deviations from the Debye relaxation become marked at millimeter-wave to sub-THz frequencies. Resonant-type contributions can clearly be seen in the far-infrared region (Fig. 2). The dominating $\tau_{1}$ process, the principal slow process for hydrogen-bond liquids, reflects the cooperative reorientational dynamics of the dipole moment $[7,8,12-15]$. Buchner, Barthel, and Stauber, combining their microwave data with FIR data of Hasted et al. [16], analyzed the dielectric spectrum of water in $0.2 \leq \nu / \mathrm{GHz} \leq 410$ at $0.2 \leq T /{ }^{\circ} \mathrm{C} \leq 35$ [12]. They showed that the dielectric spectra could be formally fitted by a superposition of two Debye processes, yielding $\tau_{1}=8.38 \mathrm{ps}$ and $\tau_{2}=1.1 \mathrm{ps}$ at $25^{\circ} \mathrm{C}$. In the present study, a more generalized model-independent approach for the high-frequency part of the spectrum has been attempted by subtracting the well-defined $\tau_{1}$-relaxation contribution [shaded area in Fig. 2(a)] from the total spectrum. This procedure revealed that dispersion $\left(\epsilon^{\prime}\right)$ and loss $\left(\epsilon^{\prime \prime}\right)$ curves clearly exhibit the features of a relaxation process in the millimeter wave to sub- $\mathrm{THz}$ frequency range.

At low frequencies, the shape of the Raman spectrum of water [Fig. 2(b)] also shows the characteristic features of relaxation as already suggested in Ref. [17]. At higher frequencies, very broad intermolecular vibration bands

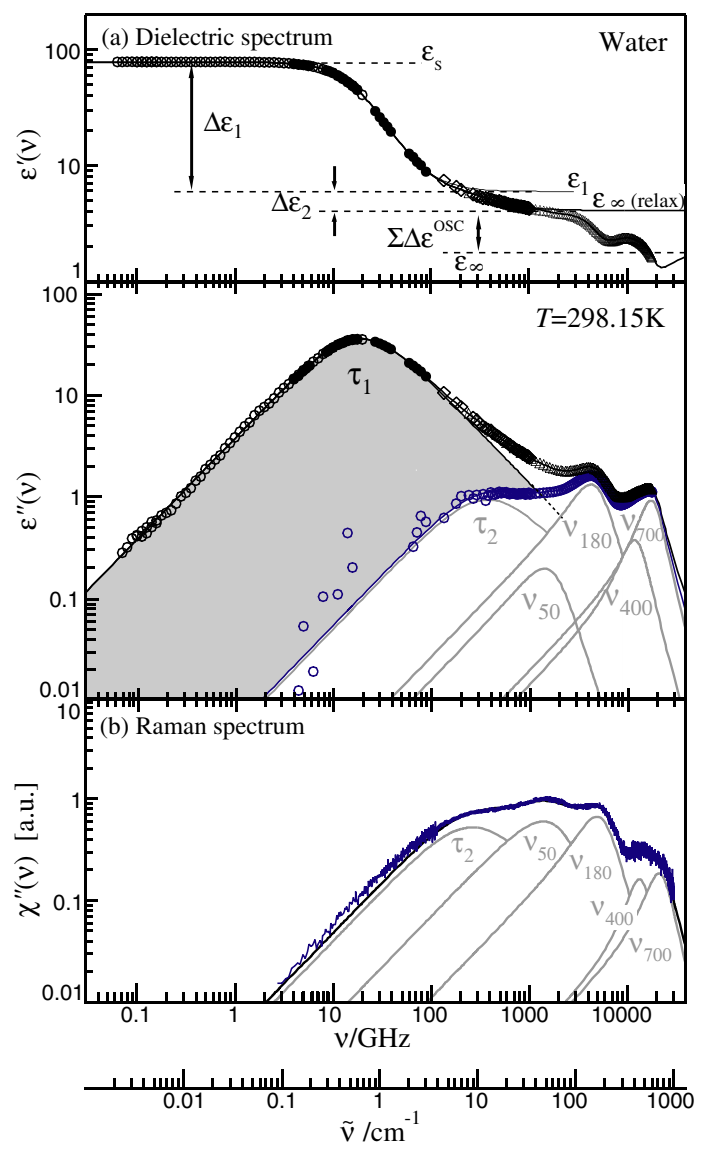

FIG. 2 (color online). (a) The complex dielectric spectrum of water in $50 \mathrm{MHz} \leq \nu \leq 18 \mathrm{THz}$ at $25^{\circ} \mathrm{C}$. The blue symbols (O) represent the residual dielectric loss after subtracting the contribution of the $\tau_{1}$ process. (b) Raman spectrum of water in $0.08 \leq \tilde{\nu} / \mathrm{cm}^{-1} \leq 1000$ at $25^{\circ} \mathrm{C}$.

are observed. Surprisingly, we found (Fig. 2) that the shape of the Raman spectrum strongly resembles the highfrequency part of the dielectric spectrum. This observation not only confirms the correspondence between these spectra for intermolecular vibration and libration bands but strongly suggests that the dielectric $\tau_{2}$-relaxation and Raman relaxation modes originate in the common molecular dynamics.

Simultaneously, we attempted a conventional approach to quantitative description by testing various relaxation models with a least-squares fitting procedure. First, we carefully checked the maximum frequency up to which the dielectric spectrum can be reproduced within the framework of relaxation models by monitoring variance and residuals of the fit. Then resonant-type functions were introduced for the higher-frequency part. The two-Debye relaxation model gives an excellent fit up to $\sim 400 \mathrm{GHz}$. Above that, the variance of the fit monitored at each $\nu$ shows a rapid linearlike increase (Fig. 3). The residuals $\delta \epsilon(\nu)=\epsilon(\nu)_{\text {data }}-\epsilon(\nu)_{\text {fit }}$ become systematically negative for $\epsilon^{\prime}(\nu)$ and positive for $\epsilon^{\prime \prime}(\nu)$, indicative of additional high-frequency dispersions. The superposition 
of two relaxation processes with $\tau_{1}=8.32 \mathrm{ps}$ and $\tau_{2}=$ $0.42 \mathrm{ps}$ and four damped harmonic oscillators centered at $\sim 60,170,420$, and $620 \mathrm{~cm}^{-1}$ well reproduces the water spectrum between $50 \mathrm{MHz}$ and $18 \mathrm{THz}$ (Fig. 2). As anticipated from the shape of the Raman spectrum in $0.08 \leq$ $\tilde{\nu} / \mathrm{cm}^{-1} \leq 1000$, a single relaxation comparable with the dielectric $\tau_{2}$ process plus four oscillators is sufficient.

In the case of methanol [Fig. 3(a)], the deviation of the dielectric spectrum from the relaxation model (a threeDebye fit [8]) becomes marked above $\nu \sim 290 \mathrm{GHz}$. The superposition of three relaxation processes (relaxation times $\tau_{1}=51.8 \mathrm{ps}, \tau_{2}=8.04 \mathrm{ps}$, and $\left.\tau_{3}=0.89 \mathrm{ps}\right)$ and two oscillators centered at $\sim 55$ and $125 \mathrm{~cm}^{-1}$ best reproduces the spectrum in $50 \mathrm{MHz} \leq \nu \leq 5 \mathrm{THz}$. The striking finding is that the low-frequency Raman spectrum of methanol [Fig. 3(b)] is also directly linked with the highfrequency deviation of its dielectric spectrum from the cooperative $\tau_{1}$ dispersion. If a model based on the superposition of relaxation and oscillator functions is applied to the Raman spectrum, two relaxation processes with $\tau_{2(\text { Raman })}=2.5 \mathrm{ps}$ and $\tau_{3(\text { Raman })}=0.55 \mathrm{ps}$ and three oscil-

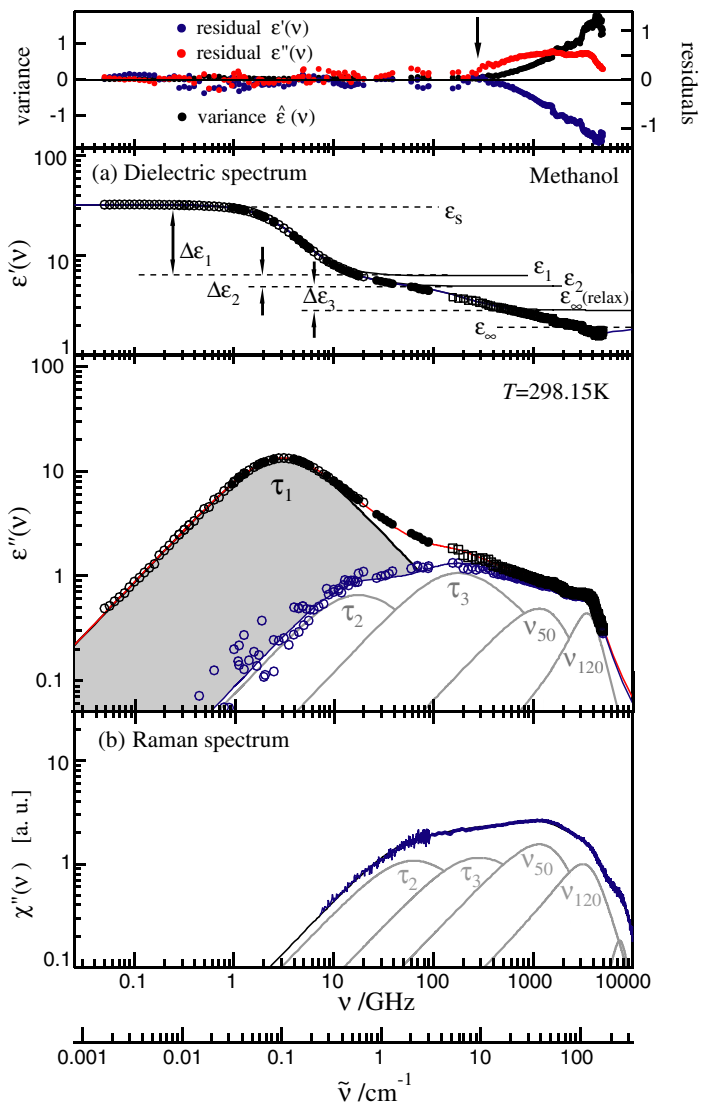

FIG. 3 (color online). (a) The complex dielectric spectrum of methanol in $50 \mathrm{MHz} \leq \nu \leq 5 \mathrm{THz}$ at $25^{\circ} \mathrm{C}$. The upper panel shows the residuals and the variance of the three-Debye fit at each $\nu$. The blue symbols $(\bigcirc)$ represent the residual dielectric loss after subtracting the contribution of the $\tau_{1}$ process. (b) Raman spectrum of methanol at $25^{\circ} \mathrm{C}$ in $0.24 \leq$ $\tilde{\nu} / \mathrm{cm}^{-1} \leq 500$. lators at $\sim 60,120$, and $260 \mathrm{~cm}^{-1}$ are required. This again confirms the relation between the low-frequency Raman spectrum and the high-frequency part of the dielectric spectrum (the $260 \mathrm{~cm}^{-1}$ band, resolved as a shoulder in Raman, is outside the frequency range of the dielectric data). The slower Raman relaxation time, which we call $\tau_{2 \text { (Raman) }}$ to emphasize the correspondence between dielectric and Raman spectra, is by a factor of about 3 shorter than the corresponding dielectric $\tau_{2}$. This implies that both processes reflect the rotational diffusion of individual methanol molecules [1] and supports previous assignments of the intermediate dielectric relaxation process of monohydric alcohols to the reorientation of individual molecules [8]. The faster Raman relaxation time $\tau_{3(\operatorname{Raman})}$ and the fastest dielectric relaxation time $\tau_{3}$ differ by a factor of less than 2. This may indicate that they are not related to diffusive motion but reflect other microscopic dynamics.

Generally, insufficient frequency coverage in the $\mathrm{THz}$ region leads to an overestimation of the fast relaxation time with $\tau_{2} \sim 1 \mathrm{ps}[8,12]$. On the other hand, data restricted to $0.1 \leq \nu / \mathrm{THz} \leq 2$ yielded $\tau_{2} \sim 0.1-0.2$ ps for water [3]. Similar values, $\tau_{2}=0.18 \mathrm{ps}$ for water as well as $\tau_{2}=$ $1.25 \mathrm{ps}$ and $\tau_{3}=0.16 \mathrm{ps}$ for methanol, were obtained by Kindt and Schmuttenmaer [18] from the power absorption spectrum $\alpha(\nu)$ in $0.9 \leq \nu / \mathrm{THz} \leq 1$.8. However, if we fix in the analysis of our $\hat{\epsilon}(\nu)$ spectra $\tau_{2}$ and/or $\tau_{3}$ to the above literature values, the variance of the fit around the loss peak frequency, $\sim 1 / 2 \pi \tau_{1}$, gets considerably worse and additional systematic deviations emerge around $300 \mathrm{GHz}$. We point out that, as we did and show in Fig. 3, it is necessary to check carefully the maximum frequency where the spectrum can be explained within the framework of relaxation models, not observing systematic deviations of the variance. We consider that the rather short $\tau_{2}$ relaxation times obtained in previous $\mathrm{THz}$ studies simultaneously reflect the lack of low-frequency data and the onset of resonant contributions.

The dielectric spectrum of ethylene glycol in $0.02 \leq$ $\nu / \mathrm{GHz} \leq 89$ (Fig. 4) can be interpreted analogously to that for methanol, resulting in three relaxation processes with $\tau_{1}=122 \mathrm{ps}, \quad \tau_{2}=21.4 \mathrm{ps}, \quad$ and $\tau_{3}=2.88 \mathrm{ps}$. Irrespective of the validity of this formal description, the high-frequency component of $\hat{\epsilon}(\nu)$, i.e., the deviation of $\hat{\epsilon}(\nu)$ from the dominating low-frequency Debye relaxation which is represented by the sum of $\tau_{2}$ and $\tau_{3}$ processes, contains information on the fast dynamics of ethylene glycol. As expected from the results for water and methanol, the feature of the low-frequency Raman spectrum of ethylene glycol also strongly resembles the high-frequency component of its dielectric spectrum. However, below $\nu \sim 5 \mathrm{GHz}$, an additional slow component can clearly be seen. Importantly, the longest Raman relaxation time $\tau_{1 \text { (Raman) }} \sim 61 \mathrm{ps}$ is far longer than $\tau_{2}$ from the dielectric data. This implies that both modes cannot be assigned to the same physical origin, but we found $\tau_{1(\text { Raman })} \approx \tau_{1} / 2$. 


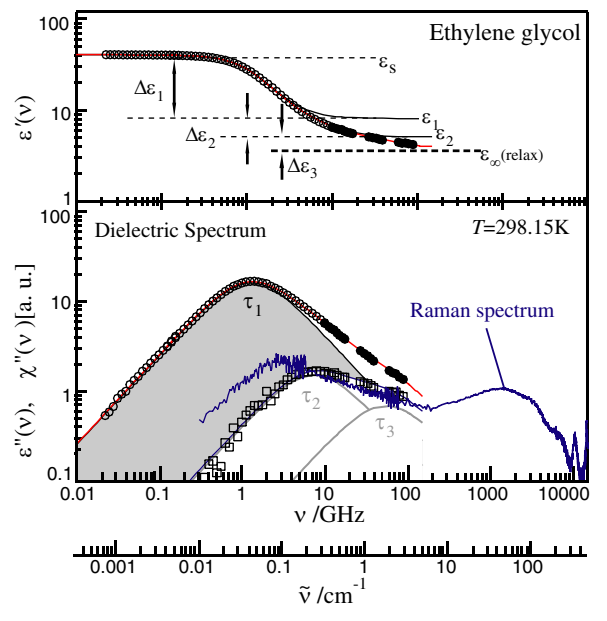

FIG. 4 (color online). The complex dielectric spectrum of ethylene glycol in $20 \mathrm{MHz} \leq \nu \leq 89 \mathrm{GHz}$ at $25^{\circ} \mathrm{C}$. The dielectric loss after subtracting the $\tau_{1}$ process (square symbols) and the Raman spectrum $\left(0.012 \leq \tilde{\nu} / \mathrm{cm}^{-1} \leq 500\right.$, blue line) are plotted in the same figure.

The relative amplitude of the slowest Raman process is considerably smaller than that of the dielectric $\tau_{1}$ process. We propose that this additional slow Raman component is a weak manifestation of cooperative relaxation that is related to the $\tau_{1}$ mode of the dielectric spectrum. Possibly, it arises from the cooperative motions of ethylene glycol conformers where the dipole moments of the $\mathrm{OH}$ groups have opposite directions and thus impose a significant quadrupole moment on the molecule.

To summarize, we have carried out a direct comparison between the dynamic susceptibilities in the frequency domain obtained from broadband dielectric $(l=1)$ and Raman $(l=2)$ spectroscopies. Different from nonhydrogen-bonding dipolar liquids, whose relaxation components in the dielectric and Raman spectra can be well explained by the common mechanism of the rotational diffusion, hydrogen-bond liquids exhibit considerably different features of these spectra in the lowfrequency region. A cooperative relaxation specific to hydrogen-bond liquids and arising from long range $\mathrm{H}-$ bond mediated dipole-dipole interactions gives the main contribution to the dielectric spectrum. This process is virtually not Raman active. Raman scattering selectively observes microscopic molecular dynamics that can be detected as the higher-frequency processes in dielectric spectrum. As indicated by the results of ethylene glycol, the contribution of quadrupolar species enables the cooperative slow relaxation to be Raman active, albeit with relatively weak intensity.
The authors acknowledge the generous support of Professor S. Kinoshita and thank Mr. Y. Fujii for technical help for Fabry-Perot interferometer measurements. The authors thank Dr. C. Rønne, Professor S. R. Keiding, and Professor C. A. Schmuttenmaer for providing THz-TDS data. T.F. acknowledges helpful discussions with Professor Y. Masuda. This work was partly supported by JSPS, the Grant-in-Aid for Scientific Research (C) for T.S., and the 21st Century COE Program at Waseda University from MEXT, Japan.

*Corresponding author.

Electronic address: takaaki.sato@waseda.jp

${ }^{\dagger}$ Corresponding author.

Electronic address: richard.buchner@chemie.uniregensburg.de

[1] W. A. Steele, in Advances in Chemical Physics, edited by I. Prigogine and S. A. Rice (Wiley, New York, 1976), Vol. 34, p. 1.

[2] E. W. Castner, Jr. and M. Maroncelli, J. Mol. Liq. 77, 1 (1998).

[3] C. Rønne, P.-O. Åstrand, and S. R. Keiding, Phys. Rev. Lett. 82, 2888 (1999).

[4] K. Winkler, J. Lindner, H. Bürsing, and P. Vöhringer, J. Chem. Phys. 113, 4674 (2000).

[5] M. C. Beard, W. T. Lotshaw, T. M. Korter, J. Heilweil, and D. McMorrow, J. Phys. Chem. A 108, 9348 (2004).

[6] S. M. Lindsay, M. W. Anderson, and J. R. Sandercock, Rev. Sci. Instrum. 52, 1478 (1981).

[7] T. Sato and R. Buchner, J. Phys. Chem. A 108, 5007 (2004).

[8] J. Barthel, K. Bachhuber, R. Buchner, and H. Hetzenauer, Chem. Phys. Lett. 165, 369 (1990).

[9] (a) H. Pickl, R. Buchner, and J. Barthel (unpublished); (b) H. Pickl, Ph.D. thesis, Regensburg University, Regensburg, Germany, 1997.

[10] D. S. Venables and C. A. Schmuttenmaer, J. Chem. Phys. 113, 11222 (2000).

[11] H. R. Zelsmann, J. Mol. Struct. 350, 95 (1995).

[12] R. Buchner, J. Barthel, and J. Stauber, Chem. Phys. Lett. 306, 57 (1999).

[13] I. Ohmine, J. Phys. Chem. 99, 6767 (1995).

[14] U. Kaatze, R. Behrends, and R. Pottel, J. Non-Cryst. Solids 305, 19 (2002).

[15] T. Sato and R. Buchner, J. Chem. Phys. 119, 10789 (2003).

[16] J.B. Hasted, S. K. Husain, F. A. M. Frescura, and J. R. Birch, Chem. Phys. Lett. 118, 622 (1985).

[17] K. Mizoguchi, Y. Hori, and Y. Tominaga, J. Chem. Phys. 97, 1961 (1992).

[18] J. T. Kindt and C. A. Schmuttenmaer, J. Phys. Chem. 100, 10373 (1996). 